\title{
NUMERICAL COMPUTATION OF A CERTAIN DIRICHLET SERIES ATTACHED TO SIEGEL MODULAR FORMS OF DEGREE TWO
}

\author{
NATHAN C. RYAN, NILS-PETER SKORUPPA, AND FREDRIK STRÖMBERG
}

\begin{abstract}
The Rankin convolution type Dirichlet series $D_{F, G}(s)$ of Siegel modular forms $F$ and $G$ of degree two, which was introduced by Kohnen and the second author, is computed numerically for various $F$ and $G$. In particular, we prove that the series $D_{F, G}(s)$, which shares the same functional equation and analytic behavior with the spinor $L$-functions of eigenforms of the same weight are not linear combinations of those. In order to conduct these experiments a numerical method to compute the Petersson scalar products of Jacobi Forms is developed and discussed in detail.
\end{abstract}

\section{Introduction AND STATEMENT OF RESUlts}

For cusp forms $F$ and $G$ in the space $S_{k}$ of Siegel cusp forms of degree 2 and even weight $k$ on the full Siegel modular group, let $D_{F, G}(s)$ be the Rankin convolution type Dirichlet series which was introduced in [10. It was proved [10, first theorem] that $D_{F, G}(s)$ shares the same analytic properties and the same functional equation as the spinor $L$-function $L(F, s)$ associated to a Hecke eigenform $F$ in $S_{k}$. In fact, if $G$ is in the spezialschaar, then one has [10, second theorem] $D_{F, G}(s)=$ $\left\langle F_{1} \mid G_{1}\right\rangle L(F, s)$ (see Section 2 for notation). However, it was left open whether $D_{F, G}(s)$, for interesting Hecke eigenform $11 F, G$, is related to the spinor $L$-functions of the forms in $S_{k}$. In any case it cannot in general simply satisfy the same identity as before since, for example, $D_{F, F}(s)$, for $F$ not in the Maass spezialschaar, has a simple pole at $s=k$ with residue equal to the Petersson norm of $F$, whereas $L(F, s)$ is holomorphic. In fact, a spinor $L$-function of a Hecke eigenform in $S_{k}$ has a pole if and only if $F$ belongs to the Maass spezialschaar [5].

The first interesting Hecke eigenform is in weight 20 and the space of such forms is 1-dimensional in weights 20 and 22, is 2-dimensional in weights 24 and 26 (which, remarkably, have rational Fourier coefficients and eigenvalues), is 3-dimensional in weight 28 and 4-dimensional in weight 30 . The interesting eigenforms in weights 20 up to 26 have rational Fourier coefficients (and eigenvalues), whereas the eigenforms in weight 28 and 30 are conjugate under Galois conjugation, respectively. The aim of this article is twofold. We report the results of numerical experiments which we performed to study the series $D_{F, G}$ for interesting eigenforms $F$ and $G$ in weights

Received by the editor August 12, 2010 and, in revised form, June 7, 2011.

2010 Mathematics Subject Classification. Primary 11F46, 11F66; Secondary 11F27, 11 F50.

This project was supported by the National Science Foundation under FRG Grant No. DMS0757627, the authors also made use of hardware provided by DMS-0821725.

${ }^{1}$ Following the terminology of [16] we call a cusp form interesting if it belongs to the orthogonal complement of the Maass spezialschaar. 
20 up to 30. Second, we develop and describe the necessary theoretical tools for our experiments. Necessary to the computations which we discuss in this paper is also the ability to compute Siegel modular forms, their Hecke eigenvalues and their spinor $L$-series. We do this via a Sage [13. package written by the first two authors [1] (with the aid of several people) and based on the algorithms developed in [16. The code and the data which we used for our computations can be found under 12 . The interested reader may use this data to reproduce our experiments.

Our computational results are summarized as follows.

Main Theorem. For $k=20,22,24,26,28,30$, let $F$ and $G$ denote simultaneous eigenforms of weight $k$ that are cusp forms but not in the Maass spezialschaar. Then $D_{F, G}(s)$ is not a linear combination of the spinor L-functions $L(H, s)$ where $H$ ranges over all simultaneous eigenforms (including noncusp forms) of weight $k$.

This result is interesting since, as already pointed out, the series $D_{F, G}(s)$ shares the same functional equation and $\Gamma$-factors as $L(F, s)$ and $L(G, s)$ yet seems not to be related to the usual spinor $L$-functions. Note that the theorem is proved by numerical computations. However, we made careful error estimates to ensure the validity of our result.

In fact, we actually prove more. Namely, we show in Section 4 that all the series $D_{F, G}(s)$ and $L(H, s)$, where $\{F, G\}$ ranges over all subsets with one or two elements of the interesting eigenforms of weight $k$, and where $H$ ranges over all eigenforms of weight $k$, are linearly independent.

Investigating the (numerically approximated) coefficients of the series $D_{F, G}(s)$ more closely we find:

Supplement to the Main Theorem. In the notation of the Main Theorem the coefficients of $D_{F, G}(s)$ are not multiplicative; that is, the (normalized) coefficients $a(n)$ do not satisfy $a(m) a(n)=a(m n)$ for all coprime pairs of integers $m$ and $n$.

We remark that this supplement implies that the series $D_{F, G}(s)$, for $F$ and $G$ not in the Maass spezialschaar, is not an $L$-function in the sense of Selberg, i.e., does not belong to the so-called Selberg class. Note that this is, like the Köcher-Maass series, a Dirichlet series which is naturally attached to Siegel modular forms, has a meromorphic continuation, satisfies a functional equation, but is not an $L$-function in the sense of Selberg. It would be desirable to have a deeper understanding of how these series $D_{F, G}(s)$ fit into the general framework of automorphic forms and their associated $L$-functions. In this context it might be interesting to remark that the series $D_{F, G}(s)$ (and the Köcher-Maass series) can be obtained as residues of a Rankin-Selberg type convolution $D_{F, G}\left(s_{1}, s_{2}\right)$ of two complex variables attached to Siegel modular forms of degree 2 [9, Prop. 1]. Moreover, the series $D_{F, G}\left(s_{1}, s_{2}\right)$ and its specialization provide further examples of Dirichlet series with analytic continuation and functional equations, but without Euler product, which can be naturally attached to Siegel modular forms [9, Thm. 2].

In Section 2 we summarize the theoretical framework for our experiments. In Section 3 we develop the necessary tools for our computations and discuss computational issues. In particular, in order to obtain our numerical results we have to develop methods to compute Poincaré series and Petersson scalar products of Jacobi forms. These methods are of independent interest; they are described in detail in Sections 3.1 to 3.3. In Section 4 we explain the proof of the main theorem. Finally, in Section 5 we summarize our numerical results. 


\section{BACKGROUND AND NOTATION}

The Siegel upper half-space of degree $d$ is given by the set

$$
\mathbb{H}^{d}:=\left\{Z=X+i Y \in \mathbb{C}^{d \times d}: Z^{t}=Z \text {, and } Y \text { positive definite }\right\} .
$$

A Siegel modular form $F: \mathbb{H}^{d} \rightarrow \mathbb{C}$ of degree $d>1$ is a holomorphic function invariant under the action of $\operatorname{Sp}(d, \mathbb{Z})$ on $\mathbb{H}^{d}$. In this paper we specialize to degree 2 and so we have a Fourier expansion of the form

$$
F\left(\begin{array}{cc}
\tau & z \\
z & \tau^{\prime}
\end{array}\right)=\sum_{\substack{r, n, m \in \mathbb{Z} \\
r^{2}-4 m n \leq 0 \\
n, m \geq 0}} a_{F}(n, r, m) q^{n} \zeta^{r} q^{\prime m},
$$

where $q=e^{2 \pi i \tau}, q^{\prime}=e^{2 \pi i \tau^{\prime}}$ and $\zeta=e^{2 \pi i z}\left(\tau, \tau^{\prime} \in \mathbb{H}^{1}, z \in \mathbb{C}\right)$. Such an $F$ is a cusp form if the $a_{F}(n, r, m)=0$ unless the quadratic form $[n, r, m]=n x^{2}+r x y+m y^{2}$ is strictly positive definite. We denote by $M_{k}$ and $S_{k}$ the space of Siegel modular forms and cusp forms of weight $k$ and degree 2 , respectively.

For each integer $\ell \geq 1$ there is a Hecke operator $T(\ell)$, a linear map that maps $S_{k}$ to $S_{k}$ (see [16, p. 386] for explicit formulas). We call a cusp form an eigenform if it is a common eigenform for all Hecke operators. To eigenforms we attach the spinor $L$-function as follows. If $T(\ell) F=\lambda_{F, \ell} F$, then define

$$
L(F, s):=\zeta(2 s-2 k+4) \sum_{\ell=1}^{\infty} \frac{\lambda_{F, \ell}}{\ell^{s}} .
$$

This function has a meromorphic continuation to the whole complex plane with at most a pole, which is then at $s=k$ and of order one. It satisfies the functional equation

$$
L^{*}(F, s):=(2 \pi)^{-2 s} \Gamma(s) \Gamma(s-k+2) L(F, s)=(-1)^{k} L^{*}(F, 2 k-2-s) .
$$

In some of our computations we renormalize (2) by $s \mapsto s+k-\frac{3}{2}$ so that the corresponding new functional equation expresses an invariance under $s \mapsto 1-s$ instead of $s \mapsto 2 k-2-s$.

We use $S_{k, N}$ for the space of Jacobi cusp forms of weight $k$ and index $N$ in the sense of [4, Chapter I]. Any $\phi$ in $S_{k, N}$ has a Fourier development of the form

$$
\phi(\tau, z)=\sum C_{\phi}(D, r) q^{\frac{r^{2}-D}{4 N}} \zeta^{r}
$$

Here the sum is over all $N$-admissible pairs, i.e., over all pairs of integers $(D, r)$ such that $D<0$ and $D \equiv r^{2} \bmod 4 N$. Recall that $C_{\phi}(D, r)$ depends on $r$ only modulo $2 N$ and that $\phi$ is a cusp form if $C_{\phi}(D, r)=0$ unless $D<0$.

As mentioned above, for an integer $k$ we denote by $S_{k}$ the space of Siegel cusp forms of weight $k$ on the full Siegel modular group, and we use $S_{k}^{*}$ and $S_{k}^{?}$ for, respectively, the subspace of Maass cusp forms and its complement. Recall that $S_{k}^{*}$ and $S_{k}^{?}$ are invariant under all Hecke operators. The first case of nontrivial $S_{k}^{?}$ occurs for weight $k=20$, where $S_{20}^{?}$ is one-dimensional. In Table 1 we list, for $20 \leq k \leq 30$, the dimensions of various subspaces of the full space of Siegel modular forms of degree 2 and weight $k$. 
Every element $F$ in $S_{k}$ admits a Fourier-Jacobi development

$$
F\left(\begin{array}{cc}
\tau & z \\
z & \tau^{\prime}
\end{array}\right)=\sum_{N \geq 1} F_{N}(\tau, z) e^{2 \pi i N \tau^{\prime}}
$$

with elements $F_{N}$ of $S_{k, N}$, respectively.

If $F$ and $G$ are both elements of $S_{k}$ we consider the Dirichlet series

$$
D_{F, G}(s):=\zeta(2 s-2 k+4) \sum_{N \geq 1} \frac{\left\langle F_{N} \mid G_{N}\right\rangle}{N^{s}},
$$

which was introduced in [10] and which may be viewed as an analogue of the Rankin convolution for elliptic modular forms. Here $\langle\phi \mid \psi\rangle$, for Jacobi cusp forms $\phi$ and $\psi$ in $S_{k, N}$, denotes their Petersson scalar product. More precisely, we have

$$
\langle\phi \mid \psi\rangle=\int_{\mathcal{D}} \phi(\tau, z) \overline{\psi(\tau, z)} v^{k-3} e^{-4 \pi N y^{2} / v} d u d v d x d y \quad(\tau=u+i v, z=x+i y),
$$

where $\mathcal{D}$ denotes any fundamental domain for the Jacobi group $\operatorname{SL}(2, \mathbb{Z})^{J}$ acting on $\mathbb{H} \times \mathbb{C}$.

\section{Method of COMPUtation}

3.1. Petersson scalar products. In order to compute numerically the coefficients of the $L$-series $D_{F, G}$ we need a method for computing the scalar product of two Jacobi forms of the same weight $k$ and index $N$. Since $F$ and $G$ are eigenforms, we can assume that their Fourier coefficients (and hence also the Fourier coefficients of the Jacobi forms in their Fourier-Jacobi development) are algebraic numbers 2 Indeed, the space of Siegel modular forms of weight $k$ on the full modular group has a basis of forms whose coefficients are rational and such that the $\mathbb{Q}$-vector space $M$ spanned by these forms is invariant under all Hecke operators; one can take as such a basis, e.g., the isobaric monomials of weight $k$ of the suitably normalized Igusa generators. The Hecke operators have thus algebraic eigenvalues, and since they can be simultaneously diagonalized over $\mathbb{C}$, it follows that there exists a number field $K$ (of finite degree over $\mathbb{Q}$ ) such that $K \otimes_{\mathbb{Q}} M$ has a $K$-basis of Hecke eigenforms, which then have Fourier coefficients in $K$. The package [11 implements methods developed in 16 to provide these Fourier coefficients in an exact representation. It is thus reasonable to look for a method to express the Petersson scalar product of two Jacobi forms in terms of their Fourier coefficients. We propose the following method.

For an $N$-admissible pair $a=(D, r)$ denote by $P_{a}$ the $a$-th Poincaré series of $S_{k, N}$. This is the unique Jacobi cusp form which satisfies

$$
\left\langle\phi \mid P_{a}\right\rangle=C_{\phi}(a)
$$

for all forms $\phi$ in $S_{k, N}$. It is clear that the $P_{a}$ with $a$ running through all admissible pairs span the space of cusp forms. Let $n$ be the dimension of $S_{k, N}$, and let

\footnotetext{
${ }^{2}$ We are indebted to the referee for the following clarification. We are not claiming here that every eigenform can be normalized so to have algebraic numbers as Fourier coefficients. In fact, as pointed out by the referee, this would be equivalent to the statement that every common eigenspace under all Hecke operators is one-dimensional, a property which is widely believed but for which, to our knowledge, there is no reference in the literature. However, this multiplicity one property holds true within the range of our computations.
} 
$h=\{h(i)\}(1 \leq i \leq n)$ be a sequence of $n$ admissible pairs. We use $\Gamma_{h}$ for the Gram matrix of the $P_{h(i)}$ with respect to the Petersson scalar product, i.e.,

$$
\Gamma_{h}=\left(C_{P_{h(i)}}(h(j))\right) .
$$

Note that the determinant of $\Gamma_{h}$ is different from 0 if and only if the $P_{h(i)}$ form a basis of $S_{k, N}$. For a form $\phi$ in $S_{k, N}$, let

$$
\gamma_{h}(\phi)=\left(C_{\phi}(h(j)), \ldots, C_{f}(h(n))\right)^{t} .
$$

We then have the following formula for the scalar product of $F$ and $G$.

Theorem 1. Assume that the Poincaré series $P_{h(i)}(1 \leq i \leq n)$ form a basis of $S_{k, N}$. Let $\phi$ and $\psi$ be Jacobi forms in $S_{k, N}$. Then one has

$$
\langle\phi \mid \psi\rangle=\gamma_{h}(\phi)^{t} \Gamma_{h}^{-1} \overline{\gamma_{h}(\psi)}
$$

Proof. Indeed, if $x$ and $y$ are the coordinate vectors of $\phi$, respectively, $\psi$ with respect to the basis $P_{h(i)}$ we have $\langle\phi \mid \psi\rangle=x^{t} \Gamma_{h} \bar{y}$. On the other hand, we deduce from $\left\langle\phi \mid P_{h(i)}\right\rangle=C_{\phi}(h(i))$ the identity $\overline{\Gamma_{h}} x=\gamma_{h}(\phi)$ and similarly $\overline{\Gamma_{h}} y=\gamma_{h}(\psi)$. Using $\overline{\Gamma_{h}}=\Gamma_{h}^{t}$ the lemma is now immediate.

3.2. Fourier coefficients of Jacobi Poincaré series. According to Theorem 1 the computation of the $N$-th coefficient in the series $D_{F, G}$ is reduced to the numerical computation of the Fourier coefficients of the Jacobi Poincaré series $P_{\Delta, r}$ in $S_{k, N}$ where $\Delta<0$ and $r^{2} \equiv \Delta \bmod 4 N$. Explicit formulas for these coefficients were originally worked out in [8, Prop. in Sect. 2]. However, we shall use the formulas from [3, Thm. 1.4], which are stated in terms of vector-valued modular forms instead of Jacobi forms. The formulas given in [3, Thm. 1.4] are more useful for us mainly because they involve in an explicit manner the Weil representation $\rho$ associated to the lattice $L:=(\mathbb{Z},(x, y) \mapsto 2 N x y)$. Explicit formulas for the matrix coefficients of this representation have been given in [15] and will be given for arbitrary lattices and in a slightly more explicit form in [19]. This makes it possible to evaluate the coefficients of the Poincaré series without having to deal with too many iterated sums (more precisely, Gauss sums inside Kloosterman sums) as provided by the formulas in $[8]$.

To be more specific, for our computations we shall use the following formulas:

Theorem 2. We have

$$
P_{\Delta, r}(z, \tau)=\alpha_{N, k, \Delta, r} \sum_{\substack{r^{\prime}, \Delta^{\prime} \in \mathbb{Z}, \Delta<0 \\ r^{\prime 2} \equiv \Delta^{\prime} \bmod 4 N}} p_{\Delta, r}\left(\Delta^{\prime}, r^{\prime}\right) q^{\frac{r^{\prime 2}-\Delta}{4 N}} \zeta^{r^{\prime}},
$$

where the Fourier coefficients are given by

$$
\begin{aligned}
p_{\Delta, r}\left(\Delta^{\prime}, r^{\prime}\right)= & \delta_{\Delta, \Delta^{\prime}}\left(\delta_{r, r^{\prime}}+\delta_{-r, r^{\prime}}\right) \\
& +2 \pi\left|\frac{\Delta^{\prime}}{\Delta}\right|^{\frac{k}{2}-\frac{3}{4}} \sum_{c \in \mathbb{Z}, c \neq 0} H_{c}^{*}\left(r, \Delta, r^{\prime}, \Delta^{\prime}\right) J_{k-\frac{3}{2}}\left(\frac{\pi}{c N} \sqrt{\left|\Delta \Delta^{\prime}\right|}\right) .
\end{aligned}
$$

\footnotetext{
${ }^{3} \mathrm{~A}$ generalization and implementation of this method for arbitrary Weil representations will be published elsewhere [19].
} 
Here $J_{v}(x)$ is the standard J-Bessel function and

$$
H_{c}^{*}\left(r, \Delta, r^{\prime}, \Delta^{\prime}\right)=\frac{e^{-\pi i \operatorname{sgn}(c) \frac{(2 k-1)}{4}}}{|c|} \sum_{\substack{d \bmod c \\
\operatorname{gcd}(c, d)=1}} \rho\left(\left(\begin{array}{ll}
a & b \\
c & d
\end{array}\right)^{*}\right)_{\underline{r}, \underline{r}^{\prime}} e\left(-\frac{\Delta a+\Delta^{\prime} d}{4 N c}\right),
$$

where $\rho$ is the Weil representation of $\mathrm{SL}(2, \mathbb{Z})$ associated to the lattice $(\mathbb{Z},(x, y) \mapsto$ $2 N x y$ ), and where, for an integer $r$, we use $\underline{r}$ for the element $\frac{r}{2 N}+\mathbb{Z}$ in $\frac{1}{2 N} \mathbb{Z} / \mathbb{Z}$ (see Section 3.3 for an explanation of these terms, and see Theorem 3 for an explicit expression for the matrix elements of $\rho$ ), and where, for each d relatively prime to $c$, we choose an element $\left(\begin{array}{ll}a & b \\ c & d\end{array}\right)$ in $\mathrm{SL}(2, \mathbb{Z})$. Finally, one has

$$
\alpha_{N, k, \Delta, r}=\frac{(\pi|\Delta| / N)^{k-3 / 2}}{8 \sqrt{N} \Gamma(k-3 / 2)} .
$$

Remark. Note that the Fourier coefficients of the Poincaré series are real as follows, e.g., from [3, Lemma 1.13 and 1.15].

Proof of Theorem 2, According to [4, Theorem 5.1] we have a one-to-one correspondence between Jacobi forms and vector-valued modular forms. Namely, if $\phi$ is a Jacobi form in $J_{k, N}$ with Fourier coefficients $C_{\phi}(\Delta, r)$, then the application

$$
\phi \mapsto H(\phi):=\sum_{\rho \bmod N} C_{\phi}(\Delta, \rho) e_{\rho / 2 N+\mathbb{Z}}
$$

defines a bijection $H: J_{k, N} \rightarrow M_{k-\frac{1}{2}, L}$. Here, for a residue class $x$ in $\frac{1}{2 N} \mathbb{Z} / \mathbb{Z}$, we use $e_{x}$ for the element in the group ring $\mathbb{C}\left[\frac{1}{2 N} \mathbb{Z} / \mathbb{Z}\right]$ which is 1 at $x$ and 0 otherwise. Moreover, following the notations in [3, Def. 1.2, p. 18], we use $M_{k-\frac{1}{2}, L}$ for the space of vector-valued modular forms $f: \mathbb{H} \rightarrow \mathbb{C}\left[\frac{1}{2 N} \mathbb{Z} / \mathbb{Z}\right]$ which satisfy

$$
f(A \tau)=w(\tau)^{2 k-1} \rho^{*}(A, w) f(\tau)
$$

for all $(A, w) \in \operatorname{Mp}(2, \mathbb{Z})$ with $\operatorname{Mp}(2, \mathbb{Z})$ denoting the usual metaplectic cover of $\operatorname{SL}(2, \mathbb{Z})$ (see [3, sec. 1.1, p. 15]). The representation $\rho$ is the Weil representation associated to the lattice $L=(\mathbb{Z},(x, y) \mapsto 2 N x y)$, i.e., the representation of $\operatorname{Mp}(2, \mathbb{Z})$ on $\mathbb{C}\left[\frac{1}{2 N} \mathbb{Z} / \mathbb{Z}\right]$, which in terms of the standard generators for $\operatorname{Mp}(2, \mathbb{Z})$ is given by

$$
\begin{aligned}
\rho\left(\left(\begin{array}{ll}
1 & 1 \\
0 & 1
\end{array}\right), 1\right) e_{r / 2 N+\mathbb{Z}} & =e^{2 \pi i r^{2} / 4 N} e_{r / 2 N+\mathbb{Z},} \\
\rho\left(\left(\begin{array}{cc}
0 & -1 \\
1 & 0
\end{array}\right), \sqrt{\tau}\right) e_{r / 2 N+\mathbb{Z}} & =\frac{e^{-2 \pi i / 8}}{\sqrt{2 N}} \sum_{r^{\prime} \bmod 2 N} e^{-2 \pi i r r^{\prime} / 2 N} e_{r^{\prime} / 2 N+\mathbb{Z} .}
\end{aligned}
$$

Finally, $\rho^{*}$ is the representation obtained from $\rho$ by taking the complex conjugates of the $\rho(A, w)$ (where we view $\rho(A, w)$ as a matrix).

By [4, Thm. 5.3] we have

$$
\langle\phi \mid \psi\rangle=\frac{1}{4 \sqrt{N}}\langle H(\phi) \mid H(\psi)\rangle,
$$

where the scalar product of two vector-valued modular forms $f_{1}$ and $f_{2}$ in $M_{k-\frac{1}{2}, L}$ is defined as [3, eq. (1.17), p. 22]:

$$
\left\langle f_{1} \mid f_{2}\right\rangle=\sum_{r \in \frac{1}{2 N} \mathbb{Z} / \mathbb{Z}} \int_{\mathrm{SL}(2, \mathbb{Z}) \backslash \mathbb{H}} f_{1, j}(\tau) \overline{f_{2, j}(\tau)} v^{k-\frac{5}{2}} d u d v \quad\left(f_{j}=\sum_{r \in \frac{1}{2 N} \mathbb{Z} / \mathbb{Z}} f_{j, r} e_{r}\right) .
$$


(Note that [4, Thm. 5.3] contains an error: the factor $1 / \sqrt{2 m}$ in Thm. 5.3 has to be replaced by $1 / \sqrt{4 m}$ as the proof in 4 actually shows.) In addition, one has to use that a fundamental domain for $\operatorname{SL}(2, \mathbb{Z})^{J} \backslash \mathbb{H} \times \mathbb{C}$ is given by $\{(\tau, x \tau+y): \tau \in$ $D, x, y \geq 0, x+y \leq 1\}$. Here $D$ is a fundamental domain for $\operatorname{SL}(2, \mathbb{Z}) \backslash \mathbb{H}$. Note the condition $x+y \leq 1$, which is due to the action of the scalar matrix -1 in $\operatorname{SL}(2, \mathbb{Z})$, which gives $(-1)(\tau, z)=(\tau,-z)$. This gives another factor of $\frac{1}{2}$, so that the correct factor in Thm. 5.3 becomes in fact $1 / 4 \sqrt{m}$ (instead of $1 / \sqrt{2 m}$ ).

From (6) and [3, Prop. 1.5, p. 23] it follows that

$$
H\left(P_{\Delta, r}\right)=\alpha_{N, k, \Delta, r} P_{r / 2 N+\mathbb{Z},|\Delta| / 4 N}^{L},
$$

where $P_{r / 2 N+\mathbb{Z},|\Delta| / 4 N}^{L}$ is the Poincaré series as introduced in [3, Eq. (1.11), p. 19]. The Fourier expansion of $P_{r / 2 N+\mathbb{Z},|\Delta| / 4 N}^{L}$ has been computed in [3, Thm. 1.4, p. 19]. The theorem is now an immediate consequence of the formula loc. cit.

3.3. The Weil representation. The last requirement to make our formulas for the Petersson scalar products of Jacobi forms explicit and ready for implementation is the determination of the matrix elements of the Weil representation $\rho$ which is defined by the formulas (5). Explicit formulas are given e.g. in [14, Prop. 1.6]. However, a direct implementation of these formulas would involve, for $A=\left(\begin{array}{ll}a & b \\ c & d\end{array}\right)$ with $c \neq 0$, a sum of length $|c|$. A trivial estimate shows that with this formula the number of exponential evaluations necessary to compute $p_{\Delta, r}\left(\Delta^{\prime}, r^{\prime}\right)$ is of the order of magnitude $M^{3}$ if we truncate the outer sum (5) at $|c|=M$. The formula of Theorem 3 below which we will use is computationally less expensive since it requires only one exponential evaluation instead of a sum of $|c|$ such evaluations.

For stating our formula we need some notation. To each matrix $A=\left(\begin{array}{ll}a & b \\ c & d\end{array}\right)$ in $\operatorname{SL}(2, \mathbb{Z})$ we associate an element $A^{*}$ in $\operatorname{Mp}(2, \mathbb{Z})$ by setting $A^{*}:=(A, \sqrt{c \tau+d})$. (Note that $A \mapsto A^{*}$ does not define a homomorphism of groups.) Here and in the sequel, for a complex number $w$, we use $\sqrt{w}$ for its root which is situated in the right half-plane but not on the negative imaginary axes. We write $e^{n}(w)$ and $e_{n}(w)$ for $e^{2 \pi i n w}$ and $e^{2 \pi i w / n}$, respectively. For $h, h^{\prime}$ in $\frac{1}{2 N} \mathbb{Z} / \mathbb{Z}$ we define the matrix element $\rho\left(A^{*}\right)_{h, h^{\prime}}$ by the equation

$$
\rho\left(A^{*}\right) e_{h^{\prime}}=\sum_{h \in \frac{1}{2 N} \mathbb{Z} / \mathbb{Z}} \rho\left(A^{*}\right)_{h, h^{\prime}} e_{h} .
$$

Finally, for a nonzero rational number $r=\frac{b}{a}(\operatorname{gcd}(a, b)=1, a>0)$ we define signature $(r)$ as the integer modulo 8 such that

$$
e_{8}(\operatorname{signature}(r))=\left(\frac{b}{a}\right) e_{8}\left(1-a_{1}+\operatorname{even}(a) b a_{1}\right),
$$

where even $(a)$ equals 1 or 0 accordingly as $a$ is even or odd, where we write $a=2^{n} a_{1}$ with odd $a_{1}$, and where $\left(\frac{b}{a}\right)$ denotes the generalized Legendre symbol (which is multiplicative in $a$ and in $b$, and which satisfies $\left(\frac{b}{2}\right)=1$ if $b \equiv \pm 1 \bmod 8$ and $\left(\frac{b}{2}\right)=-1$ if $b \equiv \pm 3 \bmod 8$.

Lemma. Let $r$ be a nonzero rational number, a its (positive) denominator, and suppose that a is not exactly divisible by 2 . Then

$$
\frac{1}{\sqrt{\tilde{a}}} \sum_{x \bmod \tilde{a}} e\left(r x^{2}\right)=e_{8}(\operatorname{signature}(2 r)),
$$


where $\tilde{a}=a / \operatorname{gcd}(2, a)$.

Proof. This identity can be easily verified using the well-known formulas

$$
\sum_{x \bmod a} e_{a}\left(b x^{2}\right)= \begin{cases}\left(\frac{b}{a}\right) \sqrt{a} & \text { if } a \equiv 1 \bmod 4, \\ \left(\frac{b}{a}\right) i \sqrt{a} & \text { if } a \equiv 3 \bmod 4, \\ \left(\frac{b}{2 a}\right) e^{2 \pi i b / 8} \sqrt{2 a} & \text { if } a=2^{n}, n \geq 2 .\end{cases}
$$

for standard Gauss sums [7, and substituting $x=2^{n} t+a_{1} u$ in a Gauss sum for a general $a=2^{n} a_{1}$ (with odd $a_{1}$ ), where $t$ and $u$ run through a complete set of representatives modulo $a_{1}$ and $2^{n}$, respectively.

Theorem 3. For $A=\left(\begin{array}{ll}a & b \\ c & d\end{array}\right)$ in $\mathrm{SL}(2, \mathbb{Z})$ and integers $x, x^{\prime}$, the matrix element $\rho\left(A^{*}\right)_{h, h^{\prime}}\left(h=x / 2 N+\mathbb{Z}, h^{\prime}=x^{\prime} / 2 N+\mathbb{Z}\right)$ of the representation $\rho$ of $\operatorname{Mp}(2, \mathbb{Z})$ defined by (5) is given by the following formula:

$$
\rho\left(A^{*}\right)_{h, h^{\prime}}=\chi(A) \sqrt{\frac{\operatorname{gcd}(2 N, c)}{2 N}} e_{4 N}\left(a b x^{2}+2 b c x y+c d y^{2}+2 z_{c}(b x+d y)+a b z_{c}^{2}\right)
$$

if there exists a $y$ such that $x^{\prime} \equiv a x+c y+z_{c} \bmod 2 N$ (and then the right-hand of this formula does not depend on the choice of $y)$, and $\rho\left(A^{*}\right)_{h, h^{\prime}}=0$ otherwise. Here $z_{c}=N$ if $c / 2 N$ is a 2-adic unit, and $z_{c}=0$ otherwise. Finally,

$$
\chi(A)=e_{8}(-\operatorname{sign}(c)) \cdot \begin{cases}e_{8}(\operatorname{signature}(a N / 2 c)) & \text { if } c / 2 N \text { is a 2-adic unit, } \\ e_{8}(\operatorname{signature}(2 a N / c)) & \text { otherwise, }\end{cases}
$$

if $c \neq 0$ and $\chi(A)=1 / \sqrt{\operatorname{sign}(d)}$ otherwise.

Proof. The claimed formula is proved in [15, Sec. 1.4] (see formulas (26), (26)' and (27) on p. 37 loc. cit.), and in a slightly different form in [18, Prop. 4.1]. Note that the formulas in [15] refer to a right action $\left.(\vartheta, \alpha) \mapsto \vartheta\right|_{\frac{1}{2}, N} \alpha$ of $\operatorname{Mp}(2, \mathbb{Z})$ on a certain space $T h_{N}$ spanned by theta functions $\vartheta_{N, \rho}(\rho \bmod 2 N)$. The precise definitions of these objects is not important here. For applying the formulas in 15. one has to identify $\mathbb{C}\left[\frac{1}{2 N} \mathbb{Z} / \mathbb{Z}\right]$ with the dual of the space $T h_{N}$ via $e_{h}\left(\vartheta_{N, \rho}\right)=1$ if $h=\rho / 2 N+\mathbb{Z}$ and $e_{h}\left(\vartheta_{N, \rho}\right)=0$ otherwise. It is then easily verified (using [15. Satz 0.1, p. 10]) that $\left(\rho(\alpha) e_{h}\right)(\vartheta)=e_{h}\left(\left.\vartheta\right|_{\frac{1}{2}, N} \alpha\right)$ for all $h$ in $\frac{1}{2 N} \mathbb{Z} / \mathbb{Z}$ and all $\vartheta$ in $T h_{M}$. Inserting for $\left.\vartheta\right|_{\frac{1}{2}, N} \alpha$ the formulas (26), (26)' and (27) of [15, Sec. 1.4] proves the claimed formulas apart from the fact that the quantity $\chi(A)$ is given in [15] in the form

$$
\chi(A)= \begin{cases}1 / \sqrt{\operatorname{sign}(d)} & c=0 \\ \frac{1}{\sqrt{\operatorname{gcd}(2 N, c) c i}} \sum_{\nu \bmod 2 c} e_{4 c}\left(a N \nu^{2}\right) & \text { if } c / 2 N \text { is a 2-adic unit } \\ \frac{1}{\sqrt{\operatorname{gcd}(2 N, c) c i}} \sum_{\nu \bmod c} e_{c}\left(a N \nu^{2}\right) & \text { otherwise. }\end{cases}
$$

(Note that formula (27) contains a typo stating that $\chi(a)=1$ for $c=0$, which, however, can be easily corrected by noticing that $\left.\vartheta_{N, \rho}\right|_{\frac{1}{2}, N}\left(\begin{array}{cc}-1 & 0 \\ 0 & -1\end{array}\right)=i^{-1} \vartheta_{N,-\rho}$. $)$ By the lemma we can rewrite $\chi(A)$ in the given form. This proves the theorem.

3.4. Implementation. All the computations were done using Sage 13 . The routines for interval arithmetic are implemented in Sage via the package mpmath. The code for computing the Weil representation, the Poincaré series and finally the files containing the necessary Gram matrices $\Gamma_{h}$ can be found on [12. The numerical computation of the Dirichlet series $D_{F, G}(s)$, which needs, in addition to the Gram 
matrices, the Fourier coefficients of the eigenforms in question is done via a short Sage script (also to be found loc. cit.), which in turn calls the Siegel modular forms package 11] mentioned in Section 1 .

3.5. Discussion of error estimates. For the computation of the Gram matrix $\Gamma_{h}$ of Theorem 11 we need to compute the Fourier coefficients $p_{\Delta, r}\left(\Delta^{\prime}, r^{\prime}\right)$ of Theorem 2 . Using the standard asymptotics of the J-Bessel function $J_{v}(x) \sim \frac{1}{\Gamma(v+1)}\left(\frac{x}{2}\right)^{v+1}$ for $x \ll 1$ and the fact that $\left|\rho(M)_{h, h^{\prime}}\right| \leq 1$ it follows that in order to obtain an error of order $\varepsilon=10^{-T}$ for the computation of a coefficient we need to truncate the sum over $c$ at about $c=M(T)$ with

$$
M(T)>O(1) 10^{\frac{T}{k-\frac{3}{2}}}
$$

with a constant (which can be made explicit) depending on $k, \Delta, \Delta^{\prime}$ and $N$. From this we see that for small values of $k$, in particular, this evaluation can be very time consuming while for higher weight $k$ we can truncate the sum earlier. At this point one should also mention that the dimension of the space $S_{k, N}$, i.e., the number of necessary Poincaré series to consider, increases linearly in $k$ and in $N$ (see [17, Thm. 1] for the dimension formula), so that the number of coefficients $p_{\Delta, r}\left(\Delta^{\prime}, r^{\prime}\right)$ to compute grows quadratically in $k$ and in $N$. In Table 2 we list the dimensions of the spaces of Jacobi cusp forms $S_{k, N}$ which fall within the range of our computations.

We need to make sure that the set of Poincaré series $\left\{P_{h(i)}\right\}_{i=1, \ldots, n}$ for a given sequence of indices $h=\left\{\left(\Delta_{i}, r_{i}\right)\right\}_{i}\left(n=\operatorname{dim} S_{k, N}\right)$ form a basis of $S_{k, N}$. For this it suffices to verify that the associated Gram matrix $\Gamma_{h}$ (44) is invertible, i.e., that its determinant is nonzero. Let $\varepsilon=10^{-T}(T \gg 1)$ and suppose that we use a sufficiently high working precision. Let $N\left(\Gamma_{h}\right)=\left(a_{i j}\right)$ be a numerical approximation to $\Gamma_{h}$ with an error less than $\varepsilon$. By this we mean that $a_{i j}=N\left(C_{P_{h(i)}}(h(j))\right)$ and $\max _{0 \leq i, j \leq n}\left|a_{i j}-C_{P_{h(i)}}(h(j))\right|<\varepsilon$. It follows that $-\varepsilon<a_{i j}-C_{P_{h(i)}}(h(j))<\varepsilon$, or equivalently $C_{P_{h(i)}}(h(j)) \in\left(a_{i j}-\varepsilon, a_{i j}+\varepsilon\right)=: I_{i j}$ for each $i, j$. Let $I\left(\Gamma_{h}\right)$ be the interval matrix with intervals $I_{i j}$ as entries. The matrix $I\left(\Gamma_{h}\right)$ is then also an approximation to $\Gamma_{h}$ in the sense that $\Gamma_{h} \in I\left(\Gamma_{h}\right)$. By elementary properties of interval arithmetic (see e.g. [1] ) one can see that $\operatorname{det} \Gamma_{h} \in \operatorname{det} I\left(\Gamma_{h}\right)$ and it is therefore enough to verify that $0 \notin \operatorname{det} I\left(\Gamma_{h}\right)$. In practice, we start with one function $P_{h(1)}$ and the corresponding one-by-one gram matrix, $\Gamma_{h}^{(1)}$. We then choose a second function, $P_{h(2)}$, and calculate the determinant (as an interval) of the interval approximation to the partial Gram matrix of these two functions. If this interval does not contain zero we add the function $P_{h(2)}$ to the set and have a nonsingular two-by-two matrix $\Gamma_{h}^{(2)}$. This procedure then continues until we have a set of $n$ linearly independent functions and a nonsingular matrix $\Gamma_{h}$.

It is not difficult to see that if the absolute error in (the infinity norm of) of our approximation $N\left(\Gamma_{h}\right)$ of $\Gamma_{h}$ is $\varepsilon$, then the absolute error of our numerical approximation $N\left(\left\langle F_{N} \mid G_{N}\right\rangle\right)$ of $\left\langle F_{N} \mid G_{N}\right\rangle$ accordingly is also bounded by a constant (depending on $F_{N}$ and $G_{N}$ ) times $\varepsilon$. To see this, first observe that in all our applications the functions $F_{N}$ and $G_{N}$ are known exactly (if defined over a number field other than $\mathbb{Q}$ we can approximate them to arbitrarily small precision). For any $\varphi, \psi \in S_{k, N}$ let $\langle\varphi \mid \psi\rangle_{N\left(\Gamma_{h}\right)}:=\gamma_{h}(\phi)^{t} N\left(\Gamma_{h}\right) \overline{\gamma_{h}(\psi)}$ denote the inner-product 
with respect to the matrix $N\left(\Gamma_{h}\right)$. Then

$$
\begin{aligned}
\left|\langle\varphi \mid \psi\rangle-\langle\varphi \mid \psi\rangle_{\tilde{\Gamma}_{h}}\right| & =\left|\gamma_{h}(\varphi)^{t}\left(\Gamma_{h}-\tilde{\Gamma}_{h}\right) \overline{\gamma_{h}(\psi)}\right| \\
& \leq\left\|\Gamma_{h}-\tilde{\Gamma}_{h}\right\|_{\infty}\left\|\gamma_{h}(\varphi)\right\|_{2}\left\|\gamma_{h}(\psi)\right\|_{2} .
\end{aligned}
$$

Thus the absolute error depends on the $l^{2}$-norms of the vectors $\gamma_{h}(\varphi)$ and $\gamma_{h}(\psi)$. Since the numbers $N\left(\left\langle F_{N} \mid G_{N}\right\rangle\right)$ tend to grow rapidly with $N$ it makes more sense to consider, as a measure of accuracy, the number of correct digits, i.e., the relative error, instead of the absolute error. Unfortunately, we are not able to estimate $\langle\varphi \mid \psi\rangle$ from below and thus can not give a corresponding "predictive" bound for the relative error in $N\left(\left\langle F_{N} \mid G_{N}\right\rangle\right)$. However, it is clear that if

$$
\left|N\left(\left\langle F_{N} \mid G_{N}\right\rangle\right)-\left\langle F_{N} \mid G_{N}\right\rangle\right|<\varepsilon \cdot\left\|\gamma_{h}\left(F_{N}\right)\right\|_{2}\left\|\gamma_{h}\left(G_{N}\right)\right\|_{2} \leq \varepsilon \cdot K
$$

and $|\varepsilon \cdot K|<\left|N\left(\left\langle F_{N} \mid G_{N}\right\rangle\right)\right|$, then the relative error can be estimated by

$$
\frac{\left|N\left(\left\langle F_{N} \mid G_{N}\right\rangle\right)-\left\langle F_{N} \mid G_{N}\right\rangle\right|}{\left|\left\langle F_{N} \mid G_{N}\right\rangle\right|}<\frac{\varepsilon \cdot K}{|| N\left(\left\langle F_{N} \mid G_{N}\right\rangle\right)|-\varepsilon \cdot K|}
$$

and we are therefore able to give a precise a posteriori bound for the relative error. Provided that $N\left(\left\langle F_{N} \mid G_{N}\right\rangle\right)$ is nonzero (which turns out to be the case in all cases we considered so far) we are always able to go back and decrease the initial $\varepsilon$ until we have achieved any desired bound. Since rigorous, or even validated, numerics never give insurance against e.g. errors in the actual implementation we also felt compelled to apply our algorithms to a case where we know the results explicitly.

Recall that if $G$ is in the Maass spezialschaar and $F$ is an eigenform, then $D_{F, G}(s)=\left\langle F_{1} \mid G_{1}\right\rangle L(F, s) L(F, s)$. To verify that the Gram data is correct as well as that the relative error behaves well, we compare the computed values for the coefficients of $D_{F, G}(s)$ with those of $L(F, s)$ (see Tables 3 and 5 ). The coefficients of $L(F, s)$ can be computed exactly using [11, It seems that in most cases the relative error can be estimated from above by a constant (not growing very fast with respect to $N)$ times $\left\|\Gamma_{h}-\tilde{\Gamma}_{h}\right\|_{\infty}$. That is, the quotient of $N\left(\left\langle F_{N} \mid G_{N}\right\rangle\right)$ divided by $\left\|\gamma_{h}\left(F_{N}\right)\right\|_{2}\left\|\gamma_{h}\left(G_{N}\right)\right\|_{2}$ does not seem to tend to zero too fast with $N$.

Another way we verify that the Gram data is correct is we check, as in [6], that the series $D_{F G}$ for $F$ and $G$ not in spezialschaar, satisfies the functional equation shown in [10].

3.6. Runtimes. To give a flavor of the amount of time needed to run an actual computation of the Gram matrices we can consider weight $k=30$ where we spent slightly less than $4 \mathrm{~h}(14254 \mathrm{~s})$ on a $2666 \mathrm{MHz}$ processor to compute the Gram matrices for $1 \leq N \leq 20$ with an error bound for the Poincaré series of $10^{-50}$. This involved computing a total of 16340 Fourier coefficients. We note that the time for a single Poincaré series computation to a given precision is essentially bounded by a constant independent of $k$ and $N$. Thus the runtime only increases with the number of coefficients required. The dependence of the runtime on the precision requested is slightly more complicated. For the proof of the Main Theorem in the case $k=30$ we needed a precision $10^{-90}$ and the corresponding Poincare series computations (with a working precision of 631 digits) took more than two weeks on the same system as mentioned above. 


\section{Proof of the Main Theorem and its supplement}

Fix a weight $k$ (between 20 and 30 ), and let $F_{1}, \ldots, F_{d}$ be a basis of common Hecke eigenforms of the full space $M_{k}$ of Siegel modular forms of weight $k$. We assume that $F_{1}, \ldots, F_{h}$ are the interesting ones, i.e., that they span the space $S_{k}$. We want to show that the series $D_{F_{i}, F_{j}}(s)$ and $L\left(F_{l}, s\right)(1 \leq i \leq j \leq h, 1 \leq l \leq d)$ are linearly independent. For this we set

$$
M:=\left(\begin{array}{cccc}
\left\langle F_{1,1} \mid F_{1,1}\right\rangle / \gamma_{1,1} & \ldots & \left\langle F_{1, n} \mid F_{1, n}\right\rangle / \gamma_{1,1} \\
\left\langle F_{1,1} \mid F_{2,1}\right\rangle / \gamma_{1,2} & \ldots & \left\langle F_{1, n} \mid F_{2, n}\right\rangle / \gamma_{1,2} \\
\vdots & & \vdots \\
\left\langle F_{e, 1} \mid F_{e, 1}\right\rangle / \gamma_{e, e} & \ldots & \left\langle F_{e, n} \mid F_{e, n}\right\rangle / \gamma_{e, e} \\
\lambda_{F_{1,1}} & \ldots & \lambda_{F_{1, n}} \\
\vdots & & \vdots \\
\lambda_{F_{d, 1}} & \ldots & \lambda_{F_{d, n}}
\end{array}\right) .
$$

Here $n=\frac{e(e+1)}{2}+d$, we use $F_{i, N}$ for the $N$-th Fourier-Jacobi coefficient of $F_{i}$, and we use $\gamma_{i, j}=\left\langle F_{i, 1} \mid F_{j, 1}\right\rangle$. The $\lambda_{F_{j, l}}$ are the eigenvalues of the $F_{j}$ with respect to $T(l)$ (see (1)). We want to show $\operatorname{det} M \neq 0$. The eigenvalues $\lambda_{F_{j, l}}$ are totally real numbers and can be computed exactly [16. Assume we approximate them numerically by rational numbers $N\left(\lambda_{F_{j, l}}\right)$ such that

$$
\left|\lambda_{F_{j, l}}-N\left(\lambda_{F_{j, l}}\right)\right|<\epsilon .
$$

Furthermore, using the method described in Section 3 we compute rational approximations $N\left(\left(\left\langle F_{i, l} \mid F_{j, l}\right\rangle / \gamma_{i, j}\right)\right.$ such that

$$
\left|\left\langle F_{i, l} \mid F_{j, l}\right\rangle-N\left(\left\langle F_{i, l} \mid F_{j, l}\right\rangle\right)\right| /\left|\gamma_{i, j}\right|<\epsilon .
$$

(The numerical calculations show that indeed $\gamma_{i, j} \neq 0$ for all $i, j$.) If we denote by $N(M)$ the matrix obtained from $M$ by replacing the coefficients $m_{p, q}$ by their respective rational approximations $N\left(m_{p, q}\right)$ we conclude that $\|M-N(M)\|_{\infty}<\epsilon$. As in Section 3.5 we construct an interval matrix approximation to $M, I(M)$, with intervals $I_{p, q}=\left(N\left(m_{p, q}\right)-\epsilon, N\left(m_{p, q}\right)+\epsilon\right)$ as entries. Since $M \in I(M)$ we are once again able to show that the determinant of $M$ is nonzero by verifying that the determinant $D$ (which is an interval) of $I(M)$ does not contain zero. If we compute the gram matrix $\Gamma_{h}$ to the precision $\epsilon_{1}$ we obtain an upper bound $\epsilon_{2}$ for the relative error $\epsilon$ in (8) by

$$
\epsilon_{2}=\epsilon_{1} \cdot \frac{\max _{i, j}\left\|\gamma_{h}\left(F_{i, j}\right)\right\|_{2}^{2}}{\min _{i, j}\left|\gamma_{i, j}\right|}
$$

It turns out that by choosing $\epsilon_{1}$ as in Table 6 we obtained an $\epsilon_{2}$ such that the determinant of the corresponding interval matrix $I(M)$ did not contain zero. This proves the theorem.

For the actual computed values of upper and lower bounds of $\left\|\gamma_{h}\left(F_{i, j}\right)\right\|_{2}$ and $\left|\gamma_{i, j}\right|$, respectively, see Table 5. The values of $\epsilon_{2}$ obtained from these bounds, as well as the determinant $D=\left(D_{m}-D_{\delta}, D_{m}+D_{\delta}\right)$ are given in Table 6 . That zero is not contained in either of these intervals is easily checked. It should also be noted that for all our computations for a given $k$, we used a working precision set high enough to represent $D$ with an absolute error comparable to $\epsilon_{2}$. As an example we computed the Poincaré series for $k=30$ using 631 digits precision.

The supplement was proved in a similar manner, using interval arithmetic and the error bounds (17). 


\section{Concluding Remarks}

We computed $D_{F, G}(s)$ numerically following the method of Section 3 for those cusp forms $F$ and $G$ listed by name in Table 1. The Fourier coefficients of these Siegel cusp forms and the coefficients of their spinor $L$-functions can be computed exactly using the package [1] which implements the methods of computation of [16]. As additional control for our calculations, we verified that our numerical approximations of $D_{F, G}$, for $F$ as in Table 1 and for a Maass spezial form $G$, equals indeed (within our error bounds) the spinor $L$-function of $F$, as predicted by the second theorem in [10] (see also Tables 3 and 5).

Andrianov asks [2, Concl. 2, p. 114] if all Dirichlet series satisfying the functional equation (2) are linear combinations of the spinor $L$-series $L(H, s)$ where $H$ runs through the eigenforms in the full space $M_{k}$ of Siegel modular forms of weight $k$ on the full Siegel modular group. We can answer this question in the negative. Namely, we computed all the Hecke eigenforms in weights 20, 22, 24, 26, 28 and 30 and their corresponding $L$-series; in particular, we computed the $L$-series for the Eisenstein series, Klingen-Eisenstein series, Maass lifts, and interesting forms that are Hecke eigenforms. For all of the cases listed in Table 1, $D_{F, G}(s)$ was not a linear combination of spinor $L$-series.

This raises the question of the nature of the series $D_{F, G}$ for interesting forms $F$ and $G$. It is natural to ask first of all whether they are $L$-functions at all in the sense of Selberg, i.e., whether they belong to the Selberg class. In fact, they do not, since they do not possess an Euler product expansion (see the supplement to the main theorem and Table 4). If we set

$$
\widetilde{D}_{F, G}(s)=\left\langle F_{1} \mid G_{1}\right\rangle^{-1} D_{F, G}\left(s+k-\frac{3}{2}\right),
$$

the functional equation (2) becomes

$$
\widetilde{D}_{F, G}^{*}(s):=(2 \pi)^{-2 s-2 k+3} \Gamma\left(s+k-\frac{3}{2}\right) \Gamma\left(s+\frac{1}{2}\right) \widetilde{D}_{F, G}(F, s)=\widetilde{D}_{F, G}^{*}(1-s) .
$$

According to [10, Theorem 1] the function $\widetilde{D}_{F, G}(s)$, for $F$ and $G$ in Table 11, has a pole at $s=\frac{3}{2}$ since the scalar product $\left\langle F_{1} \mid G_{1}\right\rangle$ is different from 0 in all these cases. This shows again that $\widetilde{D}_{F, G}(s)$ is not in the Selberg class.

\section{TABles AND Figures}

TABle 1. Dimensions of subspaces of $M_{k}$. (KE $=$ KlingenEisenstein series, $\mathrm{MC}=$ Maass spezialschaar cusp forms, Eigenforms $=$ names of Galois orbits of interesting eigenforms following the naming conventions in [16])

\begin{tabular}{|c||lllll|}
\hline$k$ & $S_{k}$ & $\mathrm{KE}$ & $\mathrm{MC}$ & $S_{k}^{?}$ & Eigenforms \\
\hline \hline 20 & 5 & 2 & 2 & 1 & $\Upsilon_{20}$ \\
22 & 6 & 2 & 3 & 1 & $\Upsilon_{22}$ \\
24 & 8 & 3 & 3 & 2 & $\Upsilon_{24 a}, \Upsilon_{24 b}$ \\
26 & 7 & 2 & 3 & 2 & $\Upsilon_{26 a}, \Upsilon_{26 b}$ \\
28 & 10 & 3 & 4 & 3 & $\Upsilon_{28}$ \\
30 & 11 & 3 & 4 & 4 & $\Upsilon_{30}$ \\
\hline
\end{tabular}


TABLE 2. Dimensions of the spaces $S_{k, N}$ for even weights $20 \leq$ $k \leq 30$ and indices $1 \leq N \leq 20$.

\begin{tabular}{|r||rrrrrrrrrrrrrrrrrrrr|}
\hline & 1 & 2 & 3 & 4 & 5 & 6 & 7 & 8 & 9 & 10 & 11 & 12 & 13 & 14 & 15 & 16 & 17 & 18 & 19 & 20 \\
\hline \hline 20 & 2 & 4 & 5 & 6 & 8 & 9 & 11 & 12 & 13 & 15 & 17 & 17 & 20 & 21 & 22 & 23 & 26 & 26 & 29 & 29 \\
22 & 3 & 4 & 6 & 7 & 9 & 10 & 13 & 13 & 15 & 17 & 19 & 19 & 23 & 23 & 25 & 26 & 29 & 29 & 33 & 32 \\
24 & 3 & 5 & 7 & 8 & 10 & 12 & 14 & 15 & 17 & 19 & 21 & 22 & 25 & 26 & 28 & 29 & 32 & 33 & 36 & 36 \\
26 & 3 & 5 & 7 & 8 & 11 & 12 & 15 & 16 & 18 & 20 & 23 & 23 & 27 & 28 & 30 & 31 & 35 & 35 & 39 & 39 \\
28 & 4 & 6 & 8 & 10 & 12 & 14 & 17 & 18 & 20 & 23 & 25 & 26 & 30 & 31 & 33 & 35 & 38 & 39 & 43 & 43 \\
30 & 4 & 6 & 9 & 10 & 13 & 15 & 18 & 19 & 22 & 24 & 27 & 28 & 32 & 33 & 36 & 37 & 41 & 42 & 46 & 46 \\
\hline
\end{tabular}

TABle 3. For the Maass lift $G$ of the first Fourier-Jacobi coefficient $\phi$ of $\Upsilon 24 a$, we compute numerically the coefficients $a(N)$ of $D_{\Upsilon 24 a, G}(s) /\langle\phi \mid \phi\rangle$ by the method in Section 3 and we compute exactly the coefficients $b(N)$ of $L(\Upsilon 24 a, s)$ (which are integers). This is an additional check that our method of computation gives correct results since a theorem in 10 tells us that, in this case, these two Dirichlet series coincide.

\begin{tabular}{|c||c|c|}
\hline$N$ & $a(N)$ & $b(N)$ \\
\hline \hline 1 & 1.0000000000000000000000000000 & 1 \\
2 & $-5.5603200000000000000000000000 \cdot 10^{6}$ & -5560320 \\
3 & $-5.3017924680000000000000000000 \cdot 10^{10}$ & -53017924680 \\
4 & $4.3592282275840000000000000000 \cdot 10^{12}$ & 4359228227584 \\
5 & $-3.3324163624500000000000000000 \cdot 10^{13}$ & -33324163624500 \\
6 & $2.9479662695669760000000000000 \cdot 10^{17}$ & 294796626956697600 \\
7 & $8.9548405531223548000000000000 \cdot 10^{18}$ & 8954840553122354800 \\
8 & $-1.7002266475219648512000000000 \cdot 10^{20}$ & -170022664752196485120 \\
9 & $3.3581886607436193369000000000 \cdot 10^{19}$ & 33581886607436193369 \\
10 & $1.8529301348457984000000000000 \cdot 10^{20}$ & 185293013484579840000 \\
\hline
\end{tabular}


TABLE 4. The first 18 coefficients of $\widetilde{D}_{\Upsilon 24 x, \Upsilon 24 y}(s)$ (see(9)), where $x, y \in\{a, b\}$, where $\Upsilon 24 a, \Upsilon 24 b$ denote the two interesting eigenforms in weight 24 . Note that $\widetilde{D}_{\Upsilon 24 x, \Upsilon 24 y}(s)$ satisfies a functional equation with respect to $s \mapsto 1-s$. More data and this data with more accuracy are available at [12].

\begin{tabular}{|c||c|r|c|}
\hline & $\widetilde{D}_{\Upsilon 24 a, \Upsilon 24 a}(s)$ & \multicolumn{1}{|c|}{$\widetilde{D}_{\Upsilon 24 a, \Upsilon 24 b}(s)$} & $\widetilde{D}_{\Upsilon 24 b, \Upsilon 24 b}(s)$ \\
\hline \hline 1 & $1.0000000000 \ldots$ & $1.0000000000 \ldots$ & $1.0000000000 \ldots$ \\
2 & $1.2562996887 \ldots$ & $-1.6301379426 \ldots$ & $1.1807313893 \ldots$ \\
3 & $1.7810603106 \ldots$ & $2.0303021423 \ldots$ & $0.8677710052 \ldots$ \\
4 & $2.0741433142 \ldots$ & $-3.4104218951 \ldots$ & $2.8372669032 \ldots$ \\
5 & $2.8899783797 \ldots$ & $0.0053403337 \ldots$ & $2.3422724376 \ldots$ \\
6 & $2.6795441187 \ldots$ & $3.4130123501 \ldots$ & $2.5830326424 \ldots$ \\
7 & $3.6002676445 \ldots$ & $1.2502722224 \ldots$ & $2.2422068960 \ldots$ \\
8 & $3.0295878336 \ldots$ & $-0.2164387720 \ldots$ & $3.5760646077 \ldots$ \\
9 & $3.9970248055 \ldots$ & $-1.9659335003 \ldots$ & $3.2031268531 \ldots$ \\
10 & $2.9874427387 \ldots$ & $-2.2751217843 \ldots$ & $3.5506087520 \ldots$ \\
11 & $3.5420885329 \ldots$ & $-0.5800680650 \ldots$ & $2.6657794870 \ldots$ \\
12 & $2.9887639258 \ldots$ & $2.3742550783 \ldots$ & $3.3541225360 \ldots$ \\
13 & $4.7252631756 \ldots$ & $-1.4101441627 \ldots$ & $3.0042305971 \ldots$ \\
14 & $3.6881022526 \ldots$ & $2.8085205331 \ldots$ & $2.5295615640 \ldots$ \\
15 & $3.2033969064 \ldots$ & $-2.1277658831 \ldots$ & $2.9089500309 \ldots$ \\
16 & $4.8466409087 \ldots$ & $1.6172735165 \ldots$ & $5.3475003622 \ldots$ \\
17 & $5.5339720201 \ldots$ & $-2.0014367544 \ldots$ & $4.1580587188 \ldots$ \\
18 & $4.5912959032 \ldots$ & $-2.3762191782 \ldots$ & $4.6382023273 \ldots$ \\
\hline
\end{tabular}

TABLE 5. Computed upper bounds for the $l^{2}$ norms of $\gamma_{h}\left(F_{i, j}\right)$ and lower bounds for the inner products $\gamma_{i, j}=\left\langle F_{i, 1} \mid F_{j, 1}\right\rangle$. The last column contains the maximum error in the comparison between the Hecke eigenvalues $\lambda_{F_{i, j}}$ and the numerical approximations, $\tilde{\lambda}_{F_{i, j}}$, computed using the inner product of $F_{i}$ with a form from the Maass spezialschaar. In all cases $1 \leq i, j \leq n$ where $n$, given in the second column, is the dimension of the space of $L$-series which we want to show is linearly independent.

\begin{tabular}{|c||c|c|l|c|}
\hline$k$ & $n$ & $\max \left(\left\|\gamma_{h}\left(F_{i, j}\right)\right\|_{2}\right)$ & $\min \left|\gamma_{i, j}\right|$ & $\max \left|\lambda_{F_{i, j}}-\tilde{\lambda}_{F_{i, j}}\right|$ \\
\hline \hline 20 & 5 & $1.2 \cdot 10^{09}$ & $1 \cdot 10^{1}$ & $3.2 \cdot 10^{-45}$ \\
\hline 22 & 6 & $8.2 \cdot 10^{04}$ & $5 \cdot 10^{-11}$ & $9.6 \cdot 10^{-56}$ \\
\hline 24 & 11 & $7.8 \cdot 10^{09}$ & $1 \cdot 10^{-10}$ & $1.7 \cdot 10^{-55}$ \\
\hline 26 & 10 & $5.2 \cdot 10^{10}$ & $1 \cdot 10^{-12}$ & $7.5 \cdot 10^{-72}$ \\
\hline 28 & 16 & $8.8 \cdot 10^{16}$ & $1 \cdot 10^{0}$ & $2.2 \cdot 10^{-71}$ \\
\hline 30 & 21 & $2.9 \cdot 10^{12}$ & $3 \cdot 10^{-25}$ & $5.6 \cdot 10^{-96}$ \\
\hline
\end{tabular}


TABLE 6 . The values used for $\epsilon_{1}$ and the computed values of $\epsilon_{2}$ as well as the computed values of the interval determinant of $I(M)$, $D=\left(D_{m}-D_{\delta}, D_{m}+D_{\delta}\right)$.

\begin{tabular}{|c||c|c|c|c|}
\hline$k$ & $\epsilon_{1}$ & $\epsilon_{2}$ & $D_{m}$ & $D_{\delta}$ \\
\hline \hline 20 & $1 \cdot 10^{-40}$ & $7.9 \cdot 10^{-26}$ & $-7.05 \cdot 10^{054}$ & $1.26 \cdot 10^{033}$ \\
\hline 22 & $3 \cdot 10^{-50}$ & $1.2 \cdot 10^{-23}$ & $-6.63 \cdot 10^{080}$ & $8.87 \cdot 10^{060}$ \\
\hline 24 & $2 \cdot 10^{-50}$ & $2.8 \cdot 10^{-13}$ & $7.38 \cdot 10^{209}$ & $1.63 \cdot 10^{206}$ \\
\hline 26 & $7 \cdot 10^{-65}$ & $4.0 \cdot 10^{-27}$ & $-6.80 \cdot 10^{190}$ & $1.27 \cdot 10^{173}$ \\
\hline 28 & $1 \cdot 10^{-65}$ & $4.8 \cdot 10^{-33}$ & $-3.21 \cdot 10^{399}$ & $6.45 \cdot 10^{384}$ \\
\hline 30 & $1 \cdot 10^{-90}$ & $2.9 \cdot 10^{-25}$ & $1.53 \cdot 10^{607}$ & $1.13 \cdot 10^{607}$ \\
\hline
\end{tabular}

\section{REFERENCES}

1. Götz Alefeld and Jürgen Herzberger, Introduction to interval computations, Computer Science and Applied Mathematics, Academic Press Inc. [Harcourt Brace Jovanovich Publishers], New York, 1983, Translated from the German by Jon Rokne. MR733988 (85d:65001)

2. A. N. Andrianov, Euler products that correspond to Siegel's modular forms of genus 2, Uspehi Mat. Nauk 29 (1974), no. 3 (177), 43-110. MR0432552 (55:5540)

3. Jan H. Bruinier, Borcherds products on $\mathrm{O}(2, l)$ and Chern classes of Heegner divisors, Lecture Notes in Mathematics, vol. 1780, Springer-Verlag, Berlin, 2002. MR1903920 (2003h:11052)

4. Martin Eichler and Don Zagier, The theory of Jacobi forms, Progress in Mathematics, vol. 55, Birkhäuser Boston Inc., Boston, MA, 1985. MR781735 (86j:11043)

5. S. A. Evdokimov, Characterization of the Maass space of Siegel modular cusp forms of genus 2, Mat. Sb. (N.S.) 112(154) (1980), no. 1(5), 133-142, 144. MR.575936 (82a:10028)

6. David W. Farmer, Nathan C. Ryan, and Ralf Schmidt, Finding and verifying the functional equation for high degree Euler-products, Pac. J. Math. (To appear).

7. Carl-Friedrich Gauss, Summatio quarundam serierum singularium, Com Soc. Rec. Sci. Göttingensis Rec. 1 (1811).

8. B. Gross, W. Kohnen, and D. Zagier, Heegner points and derivatives of L-series. II, Math. Ann. 278 (1987), no. 1-4, 497-562. MR909238 (89i:11069)

9. Özlem Imamoḡlu and Yves Martin, On a Rankin-Selberg convolution of two variables for Siegel modular forms, Forum Math. 15 (2003), no. 4, 565-589. MR1978335 (2004k:11068)

10. W. Kohnen and N.-P. Skoruppa, A certain Dirichlet series attached to Siegel modular forms of degree two, Invent. Math. 95 (1989), no. 3, 541-558. MR979364 (90b:11050)

11. Nathan C. Ryan and N.-P. Skoruppa, Sage-add-ons: A Sage package for computing Siegel modular forms of degree 2, 2009, http://hg. countnumber.de.

12. Nathan C. Ryan, Nils-Peter Skoruppa, and Fredrik Strömberg, Data on the Rankin convolution of two Siegel modular forms of degree 2, August 2009, http://data.countnumber.de/Lfg.

13. The SAGE Group, SAGE Mathematics Software (Version 4.1), 2009.

14. T. Shintani, On construction of holomorphic cusp forms of half integral weight, Nagoya Math. J. 58 (1975), 83-126. MR0389772 (52:10603)

15. Nils-Peter Skoruppa, Über den Zusammenhang zwischen Jacobiformen und Modulformen halbganzen Gewichts, Bonner Mathematische Schriften [Bonn Mathematical Publications], 159, Universität Bonn Mathematisches Institut, Bonn, 1985, Dissertation, Rheinische FriedrichWilhelms-Universität, Bonn, 1984. MR806354 (87a:11045)

16. - Computations of Siegel modular forms of genus two, Math. Comp. 58 (1992), no. 197, 381-398. MR1106982 (92e:11041)

17. Nils-Peter Skoruppa and Don Zagier, Jacobi forms and a certain space of modular forms, Invent. Math. 94 (1988), no. 1, 113-146. MR958592(89k:11029) 
18. _ A trace formula for Jacobi forms, J. Reine Angew. Math. 393 (1989), 168-198. MR972365 (90b:11045)

19. Fredrik Strömberg, Weil representation associated to finite quadratic modules, preprint (2011), arXiv:1108.0202.

Department of Mathematics, Bucknell University, Lewisburg, Pennsylvania 17837

E-mail address: nathan.ryan@bucknell.edu

Fachbereich Mathematik, Universität Siegen, Germany

E-mail address: nils.skoruppa@uni-siegen.de

Fachbereich Mathematik, TU-Darmstadt, Germany

E-mail address: stroemberg@mathematik.tu-darmstadt.de 